\title{
The Severity of Ischemia Varies in Sprague-Dawley Rats from Different Vendors
}

\author{
Simona Nikolova, ${ }^{1}$ Ting-Yim Lee, ${ }^{1,2}$ and Robert Bartha ${ }^{1,2,3,4}$ \\ ${ }^{1}$ Imaging Research Laboratories, Robarts Research Institute, University of Western Ontario, P.O. Box 5015, \\ 100 Perth Drive, London, ON, Canada N6A 5K8 \\ ${ }^{2}$ Department of Medical Imaging, University of Western Ontario, London, ON, Canada N6A $3 K 7$ \\ ${ }^{3}$ Department of Medical Biophysics, University of Western Ontario, London, ON, Canada N6A $3 K 7$ \\ ${ }^{4}$ Department of Psychiatry, University of Western Ontario, London, ON, Canada N6A 3K7
}

Correspondence should be addressed to Robert Bartha; rbartha@robarts.ca

Received 12 November 2013; Accepted 29 December 2013; Published 9 February 2014

Academic Editors: A. Ducruet and J. Mocco

Copyright (C) 2014 Simona Nikolova et al. This is an open access article distributed under the Creative Commons Attribution License, which permits unrestricted use, distribution, and reproduction in any medium, provided the original work is properly cited.

\begin{abstract}
The purpose of this study was to compare acute cerebral perfusion measured by computed tomography, stroke lesion volume measured by magnetic resonance imaging, and motor function in Sprague-Dawley rats supplied by Charles River (Charles River, Quebec, Canada) and Harlan (Harlan, Michigan, USA). During the acute stages of ischemia ( $<3$ hours), Sprague-Dawley rats supplied by Harlan had a greater reduction in blood flow (67\%) than rats supplied by Charles River (37\%). MRI at days 1 and 6 after ischemia showed larger lesions in the Charles River animals compared to Harlan animals $(P<0.05)$ at both time points. Lesion volume decreased in both Charles River and Harlan rats at day 6 compared to day $1(P<0.05)$ and corresponded to lesion size on histology. The Harlan animals had significant functional deficits $(P<0.05)$ one day after surgery in postural hang reflex, forelimb placement, and tactile fraction first tests, whereas rats supplied by Charles River had no significant functional impairment as a result of surgery. The current study provides evidence that differences in response to ischemia between rats of the same strain supplied by different vendors should be an important consideration when animals are selected for the study of cerebral ischemia.
\end{abstract}

\section{Introduction}

Various focal ischemia models can be used [1-7] to evaluate potential therapies for stroke. These models typically involve occlusion of the middle cerebral artery (MCA) and are subdivided into permanent and reversible. Permanent models include the Tamura approach $[4,6,7]$ and its modifications involving the use of clips and threads [8-12], cauterization, [13] or reversible snare ligature [14]. Cauterization of the MCA followed by permanent occlusion of the ipsilateral carotid artery and temporary occlusion of the contralateral carotid artery is one of the most frequently used models for focal and irreversible ischemia in rats [1-5]. This model of ischemia allows only partial reperfusion and results in a reproducible predominantly cortical insult in the primary somatosensory cortex (S1FL, S1BF) and the CA1 field of the hippocampus, without severe impairment of motor function [15]. The temporary occlusion of the contralateral carotid artery reduces cerebral blood flow to ischemic ranges. This model is typically chosen because of its low mortality rate and is used for investigations of the biochemical changes during cerebral ischemia [15].

When considering the animal model for ischemia studies, several criteria must be evaluated including the animal species and strain, as well as the animal supplier. Ischemia models in mice and rats are common due to the high reproducibility, low cost, and relevance of these species. Despite the large number of genetic knockouts available in mice, rats are favourable in studies that examine the ischemic lesion using noninvasive imaging (magnetic resonance imaging (MRI), computed tomography (CT), and positron emission tomography (PET)). The larger rat brain size facilitates imaging with greater resolution and sensitivity. Previous studies have shown differences between rats of the same strain from different suppliers in response to various insults. For example, Griffin et al. [16] demonstrated large differences in 
susceptibility to neuropathy after nitric oxide (NO) inhibition in Sprague-Dawley (SD) rats supplied by Harlan (Harlan, Michigan, USA, HN) and Charles River (Charles River, Quebec, Canada, CR). Similarly, Pollock and Rekito [17] suggested that NO plays a more significant role in the maintenance of arterial pressure in $\mathrm{HN}$ rats compared to CR rats. In addition, an epilepsy study by Langer et al. [18] showed that SD rats supplied by CR have lower sensitivity to status epilepticus induction than $\mathrm{HN}$ rats. Therefore, prior to selecting rats for the study of cerebral ischemia, the nature of the study and the variations in anatomy should be carefully noted.

Although extensive research has been carried out in the field of animal models, supplier differences, and ischemic outcome $[9,19-26]$, a direct comparison of Sprague-Dawley rats from different suppliers has not been made for the cautery plus two-vessel occlusion model of ischemia. The severity of injury is highly dependent on the vascular anatomy of the rat including the presence of collateral circulation $[24,25$, 27] and also likely dependent on the method of occlusion. Therefore, the purpose of this study was to compare early changes in blood flow within the ischemic lesion measured by computed tomography (CT), lesion size measured by magnetic resonance imaging (MRI), and motor function in Sprague-Dawley rats supplied by Harlan and Charles River.

\section{Materials and Methods}

2.1. Animal Model. Twelve male Sprague-Dawley (SD) rats (6 from the CR laboratory (average weight \pm standard error $352 \pm 52 \mathrm{~g}$ ) and 6 from the HN laboratory (average weight \pm standard error $314 \pm 21 \mathrm{~g}$ )) were studied according to a protocol approved by the Animal Use Subcommittee of University of Western Ontario. Ischemia was induced by occlusion of the left MCA using cautery along with permanent ipsilateral carotid artery occlusion by double ligation, followed by a temporary (1 hour) contralateral carotid artery occlusion.

Briefly, anesthesia was induced $\left(5 \%\right.$ in air/ $\left.\mathrm{O}_{2}\right)$ and maintained $\left(2-2.5 \%\right.$ in air $\left./ \mathrm{O}_{2}\right)$ with isoflurane by way of endotracheal intubation. The middle cerebral artery (MCA) was occluded using a slightly modified version of the Tamura technique $[6,7]$. The animal was placed prone in a stereotaxic frame (Stoelting Company, Illinois, USA). A small T-shaped skin incision was made at the midpoint between the left orbit and the external auditory canal. Subsequent dissection was carried out using microsurgical techniques. The skin incision was retracted and the temporalis muscle was dissected away from the skull along the temporal line. The muscle was then retracted ventrally. A craniotomy was made at the junction between the medial wall and the roof of the inferotemporal fossa. Following the removal of the dura the MCA was occluded by cautery between its cortical branch to the rhinal cortex and the lateral striate arteries. The craniotomy was covered by a small piece of gel foam, the temporalis muscle was sutured with 1-2 stitches, the soft tissue was allowed to fall back into place, and the skin was closed using 5-7 sutures in a simple interrupted pattern. The right common carotid artery (RCC) was exposed through a midline ventral cervical incision. The RCC was isolated while preserving the vagus nerve, and a loop or 4-0 silk was placed around the artery for future manipulation. The left common carotid (LCC) artery was also exposed and permanently occluded by double ligation with 4-0 silk and a cut between the two ligatures. A 60-minute temporary occlusion of the RCC artery was performed in the CT suite after the baseline CT scan by using traction provided by fine forceps. Anesthesia was maintained by adjusting the isoflurane levels as needed $(2.5 \%-3 \%)$. At the end of the 60 minute occlusion, the incision was closed with a suture.

During the surgical procedure the rats were ventilated to normocapnia. Following surgical preparation the rats were transported to the CT and MRI facilities for imaging. Rectal temperature was maintained between 36.5 and $37.5^{\circ} \mathrm{C}$ at all times. At all times during the experiments, heart rate and $\mathrm{SPO}_{2}$ levels were monitored. Physiologic parameters in all the experiments were recorded every 10 minutes after initiation of ischemia.

2.2. CT and MR Imaging. CT perfusion scans were obtained using a General Electric (GE) micro-CT scanner [28] (eXplore Locus Ultra Preclinical in-vivo Micro CT scanner, GE Healthcare, London, ON). For CT perfusion imaging, a contrast-enhanced cine continuous CT scan was performed where the CT couch remained stationary while the gantry rotated continuously, acquiring images simultaneously from 4 different (contiguous) slice locations. A $36 \mathrm{~mm}$ slab of brain was chosen for perfusion imaging, consisting of 40 and $0.9 \mathrm{~mm}$ slices that included the brainstem, cerebellum, deep grey matter (consisting of the basal ganglia and thalamus), and parietooccipital cortex. At the beginning of each scan, $0.8 \mathrm{~mL}$ of contrast (Omnipaque 300, GE Healthcare) was injected at the rate of $8 \mathrm{~mL} / \mathrm{min}$ at the start of the acquisition of 1600 images, divided into 40 volumes of 40 slices each, over $40 \mathrm{~s}$. The image acquisition parameters were $80 \mathrm{kVp}$ and $60 \mathrm{~mA}, 512 \times 512$ image matrix, $12 \mathrm{~cm}$ field of view, and $1 \mathrm{~s}$ per rotation. Two CT scans were performed before and immediately after the temporary occlusion.

Functional maps of cerebral blood flow (CBF), cerebral blood volume (CBV), and mean transit time (MTT) were calculated with CT Perfusion software (GE Healthcare, Waukesha, WI) from the acquired perfusion imaging data sets. One $2 \times 2$ pixel region of interest was placed in the common carotid artery to obtain arterial concentration curves. $\mathrm{CBF}$ and $\mathrm{CBV}$ maps of ischemic and contralateral areas were created at three time points as follows: a day before occlusion and immediately after the one hour transient vessel occlusion.

MR measurements were made using a Varian 9.4T MR scanner (Varian Inc. Palo Alto, California). MR fast spin echo (FSE) images $(\mathrm{TE}=44 \mathrm{~ms}, \mathrm{TR}=5000 \mathrm{~ms}$, FOV $38.4 \times$ $38.4 \mathrm{~mm}^{2}, 20-2 \mathrm{~mm}$ thick slices, acquisition matrix 256 $\times 256$ ), as well as MR diffusion weighted images (DWI) $\left(\mathrm{TE}=35.09 \mathrm{~ms}, \mathrm{TR}=1000 \mathrm{~ms}, \mathrm{FOV} 38.4 \times 38.4 \mathrm{~mm}^{2}, b=\right.$ $1000 \mathrm{~s} / \mathrm{mm}^{2}, 1 \mathrm{~mm}$ thick slices, acquisition matrix $\left.128 \times 128\right)$, were obtained at day one and six days after the occlusion. Lesion volumes at day 1 were calculated by manually outlining the regions of ischemia using the ImageJ (NIH, version $1.33 \mathrm{~b}$ ) analysis software package. Changes within the ischemic region at day 6 were identified by manually outlining a region of interest that encompassed the stroke area as identified by the corresponding TTC stained sections. 
MR regions of interest outlining the lesion volume were saved and imported into the CT software where they were used as a guideline for cerebral blood flow measurements.

2.3. Behavioral Assessment. Five different behavioural tests were performed to assess the extent of neurological impairment and abnormal upper body posture in the ischemic animals. These tests were performed before occlusion (day 0 ), 24 hours after occlusion (day 1), and 6 days after the occlusion (day 6). The latter tests encompassed a range of difficulty and demonstrated sensitivity in revealing long term functional deficits compared to simple neurological scores [29-34].

2.3.1. Cylinder Test. The Schallert cylinder test was used to examine asymmetries in forelimb use for postural support behaviors [32]. This test encouraged upright support against the cylinder walls revealing forelimb asymmetries. Rats were placed in a plexiglass cylinder (diameter: $20 \mathrm{~cm}$; height: $40 \mathrm{~cm}$ ) and videotaped in the cylinder. During slow-motion video playback, instances of the sole use of the ipsilateral (to the lesion) or contralateral forelimb or the simultaneous bilateral use of both forelimbs for upright support were recorded. Asymmetry score was calculated as follows: (contralateral + $1 / 2$ bilateral)/total number of support observations, given as a function of days after lesion.

Limb adduction was defined as retraction of a forelimb for at least $2 \mathrm{~s}$ while rats were stationary, in a horizontal position resting on the remaining paws [35]. The number of contralateral adductions per $2 \mathrm{~min}$ of observation was recorded.

\subsubsection{Coordinated Forelimb Placement during Locomotion} (foot fault test). The foot fault test was used to measure coordinated forelimb placement during locomotor movements [36]. Animals were placed on an elevated grid platform $\left(33 \mathrm{~cm} \times 30 \mathrm{~cm}\right.$; grid openings: $12.25,8.4$, and $\left.6.25 \mathrm{~cm}^{2}\right)$ and were videotaped for 2 minutes as they walked on the platform. Rats moved across the platform placing their paws on the grid. Errors (foot faults) were measured as slips with either forelimb through the grid opening. Using slow-motion video playback the total number of steps and the total number of foot faults were recorded. Data were analyzed as the percentage of contralateral errors per forelimb step $(\%=$ forelimb errors/forelimb steps).

2.3.3. Bilateral Tactile Stimulation Test. The Bilateral Tactile Stimulation test (also known as the "sticky tape test") was used to measure forelimb somatosensory asymmetries $[32,37,38]$. The test measured responsiveness to tactile stimulation applied to the distal forelimbs. Circular adhesive patches (AVERY multipurpose round removable labels, $1.2 \mathrm{~cm}$ diameter) were applied to the rat forelimbs. Latencies to remove stimuli with were recorded for the stimuli on each forelimb (four to five trials per each time point). Animals with forelimb somatosensory impairments showed a bias in contacting the ipsilateral stimulus. Data were presented as the percentage of trials (four or five) in which the animal contacted the ipsilateral stimulus first. Maximum cutoff latency was considered to be 3 minutes, 3 trials/session, with 1-2 minutes intertrial interval.
2.3.4. Forelimb Placing Response for Vibrissal Stimulation. For the placing test animals were held by the torso with their forelimbs hanging freely [36]. They were then moved slowly toward the edge of the countertop until the vibrissae of one side made contact with the edge. Intact animals would typically quickly place their forelimb onto the edge of a counter top when the ipsilateral vibrissae were brushed against the table edge. In contrast, rats with unilateral lesions would present with impairments in the placing response of the limb contralateral to the lesion. This deficit was even more pronounced in conjunction with the extinction placing test. In the extinction placing test, as the vibrissae of one side were brushed against the edge of the counter top, the contralateral vibrissae were gently stimulated thus providing competing vibrissal stimulation. Animals were tested on 10 trials of each forelimb. Placing asymmetries were presented as the percentage of contralateral and ipsilateral forelimb placements per trial (number of successfully raised forelimb in 10 trials for each forelimb).

2.3.5. Postural Hang Reflex (PHR). Developed by Bederson et al., [39] this test was performed to evaluate sensorimotor function assessed by upper body posture. The degree of abnormal posture was estimated by suspending the rats with their tails $50 \mathrm{~cm}$ above the floor and slowly lowering them towards it. Abnormal posture involved flexing the contralateral limb toward the body in light impairment cases and circling towards the ipsilateral side in more severe cases.

2.4. Histology. At day 6, rat brains were extracted from the skull immediately after sacrificing the animal. Brains were placed in a cutting frame, covered with TissueTek (Sakura Finetechnical Co LTD, Tokyo, Japan), and then frozen for 10-15 min. After freezing they were sliced into $2 \mathrm{~mm}$ coronal sections. Tissue sections were incubated with $2 \% 2,3,5-$ triphenyltetrazolium chloride (TTC) for 30 minutes to identify viable and nonviable tissue [40] and then $10 \%$ buffered formalin. TTC stains viable tissue red, while tissue without viable mitochondria remains unstained (white, shown in Figure 1). TTC stained sections were scanned with an EPSON Perfection 636U flatbed scanner (EPSON, Canada Ltd., Figure 1(a)) and visually compared to the MR and CT images.

2.5. Statistical Comparisons. Comparison of behavioural outcomes were made between Charles River and Harlan animals on days 0,1 , and 6 and between animals from the same supplier on days 0,1 , and 6 after surgery. Analyses were performed using Welch's 2 tailed, unequal sample size (due to morbidity), unequal variance $t$-test. In Table 1 , significance is represented by an asterisks (*) for $P$ values less than 0.05 , and a dash (-) for $P$ values $>0.05$ (no significance).

Average stroke volume differences between one day and six days after occlusion in animals supplied by Harlan and Charles River were compared using two tailed paired $t$-tests. Average stroke volume differences between suppliers were compared using a two tailed unpaired unequal variance $t$ tests at one day after and 6 days post occlusion. 


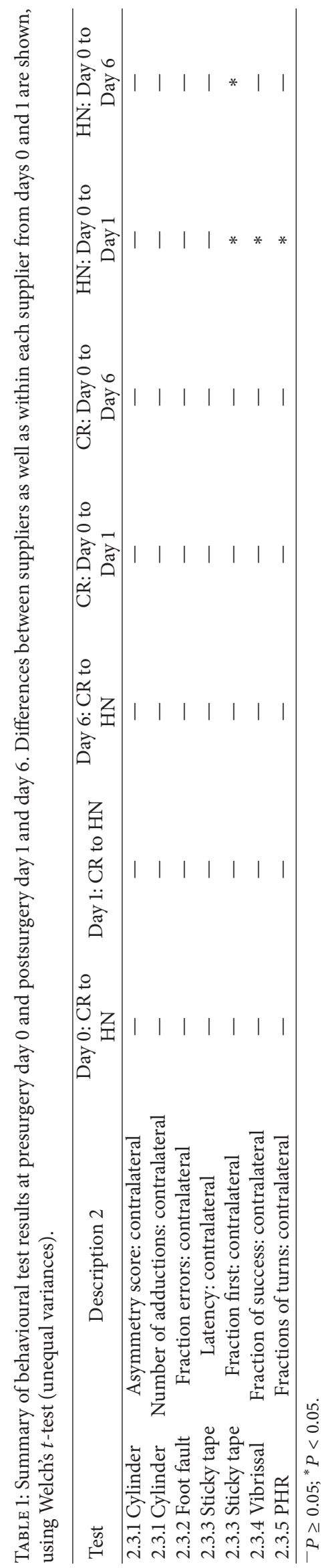


Harlan (HN)

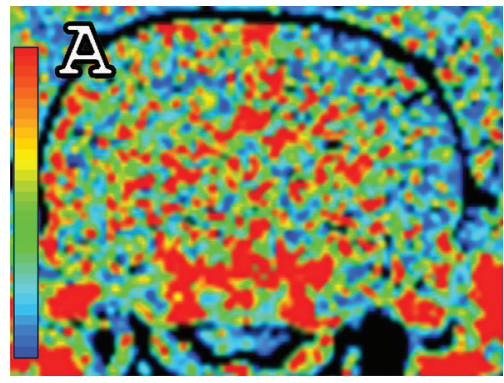

(a)

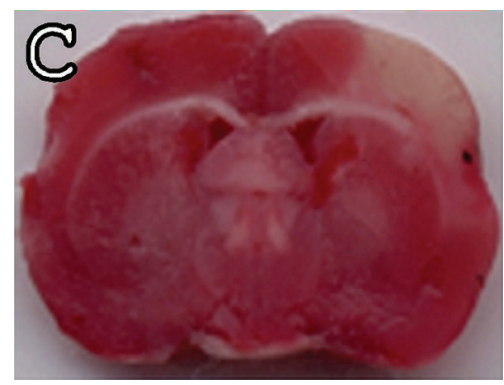

(c)

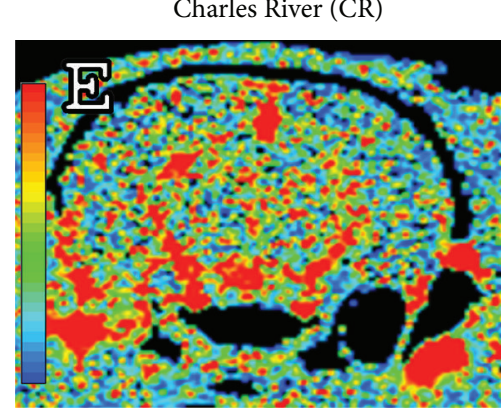

(e)

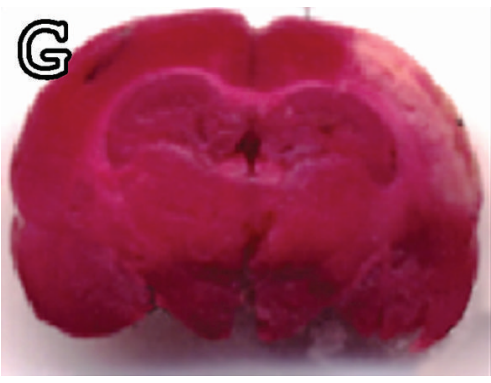

(g)
Harlan (HN)

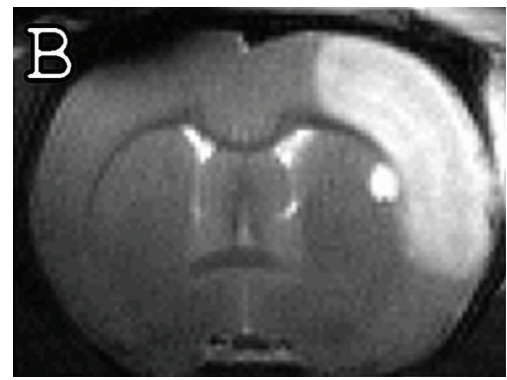

(b)

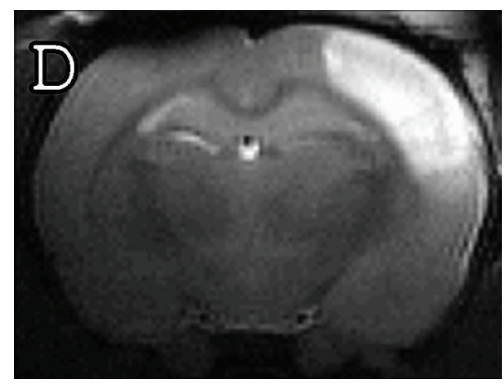

(d)

Charles River (CR)

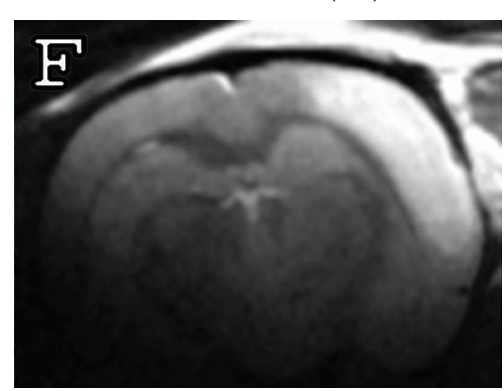

(f)

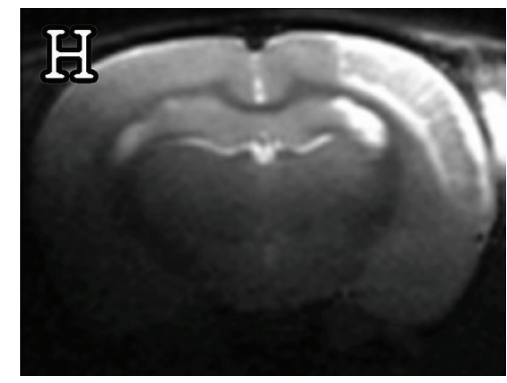

(h)

Figure 1: CT blood flow maps (color bar represents a linear scale from 0 (blue) to 1500 (red) $\mathrm{mL} / 100 \mathrm{~g} / \mathrm{min}$ ). ((a), (e)) Following permanent ipsilateral occlusion and 1 hour of transient common carotid artery occlusion along with the corresponding MRI FSE image. ((b), (f)) obtained at day 1 . The hyperintensity in the $\mathrm{T}_{2}$-weighted FSE images represents regions of tissue damage. The TTC stained histology images ((c), (g)) and the corresponding MRI FSE image $((\mathrm{d}),(\mathrm{h}))$ obtained at day 6 show final lesion size. MRI slices at the 24-hour time point were matched as closely as possible to the 6-day time point.

\section{Results}

3.1. Animal Model. Anesthesia level was kept in the range of $2-3 \%$ in all groups. Rectal temperature was maintained at $36.5-37.5^{\circ} \mathrm{C}$. At all times during the experiments, heart rate was between 330 and 380 beats per minute and $\mathrm{SPO}_{2}$ levels were in the range of $88-93 \%$.

3.2. MR and CT Imaging. CT blood flow maps following permanent ipsilateral occlusion and 1 hour of transient occlusion 
are shown in Figure 1 along with the corresponding MRI FSE images at day 1 in the HN and CR rat. The bottom row of Figure 1 shows the final TTC stained histology images and the corresponding MRI FSE images ( $\mathrm{TE}=44 \mathrm{~ms}$ ) obtained at day 6. The blue regions in the CT images indicate regions of reduced blood flow. One hour after the temporary occlusion, an average $37 \% \pm 4 \%$ decrease in blood flow was observed in the SD rats supplied by Charles River, while an average $67 \% \pm 6 \%$ decrease in blood flow was observed in the SD rats supplied by Harlan. The hyperintense pixels on the MRI correspond to regions of tissue damage, which was confirmed by TTC staining. Figure 2 shows the percent survival rate as a function of time in both animal groups. All animals from Charles River survived to day 6 while there was $50 \%$ mortality in the animals from Harlan at day 6 . Figure 3 shows ischemic damage in a representative CR animal at day 1 (top row), day 6 (middle row), and the final histology (bottom row). The average stroke volume in the CR and HN animals at day 1 is shown in Figure 4. Both HN and CR rats had significant decrease in stroke volume on day 6 compared to day $1, P=0.044$ and $P=0.001$, respectively (two tailed paired $t$-test). The stroke volume in rats supplied by Harlan $\left(162 \pm 29 \mathrm{~mm}^{3}\right)$ was significantly smaller than the stroke volume in rats supplied by Charles River $\left(335 \pm 50 \mathrm{~mm}^{3}, P=0.018\right.$, two tailed, unequal variance $t$-test). Similarly, at day six, lesion volumes in rats from Harlan $\left(73 \pm 30 \mathrm{~mm}^{3}\right)$ were significantly smaller than the lesion volume observed in rats from Charles River $(210 \pm$ $36 \mathrm{~mm}^{3}, P=0.024$, two tailed, unequal variance $t$-test).

3.3. Behavioral Assessment. Table 1 lists all behaviour tests performed and the comparison between animal groups. Function was compared between $\mathrm{CR}$ and $\mathrm{HN}$ animals at baseline (preischemia) at day 1 and day 6 after ischemia. Comparative differences from baseline for each group were made at day 1 and day 6 after stroke. Harlan rats displayed greater behavioral response to ischemia than their Charles River counterparts as shown in Table 1. Significant deficits $(P<0.05$, unpaired, 2 two-tailed $t$-test with unequal variance) in performance were measured for $\mathrm{HN}$ rats at day 1 after surgery as compared to presurgery for PHR, vibrissal, and sticky tape fraction first tests, whereas CR rats had no significant functional impairment as a result of surgery on day 1 . At day 6 , behaviour returned to baseline with only one exception for the sticky tape fraction first test for HN rats.

\section{Discussion}

Cautery in combination with two-vessel occlusion is a reproducible model of acute ischemia in the Sprague-Dawley rat [15]. The lesions were consistently located in the primary somatosensory cortex forelimb regions (S1FL), dysgranular zone (S1DZ), and parietal association area marked by barrel field (S1BF and S1ULp) and sometimes extending to the secondary somatosensory cortex (S2) and the $\mathrm{C} 1, \mathrm{G} 1$, and D1 fields in the animals supplied by CR. This model was chosen for the current study because it was reproducible and injuries were in tissue that could be functionally assessed. Cortical regions in the rat brain are heavily supplied by many collateral blood vessels $[24,25,27]$ and therefore the

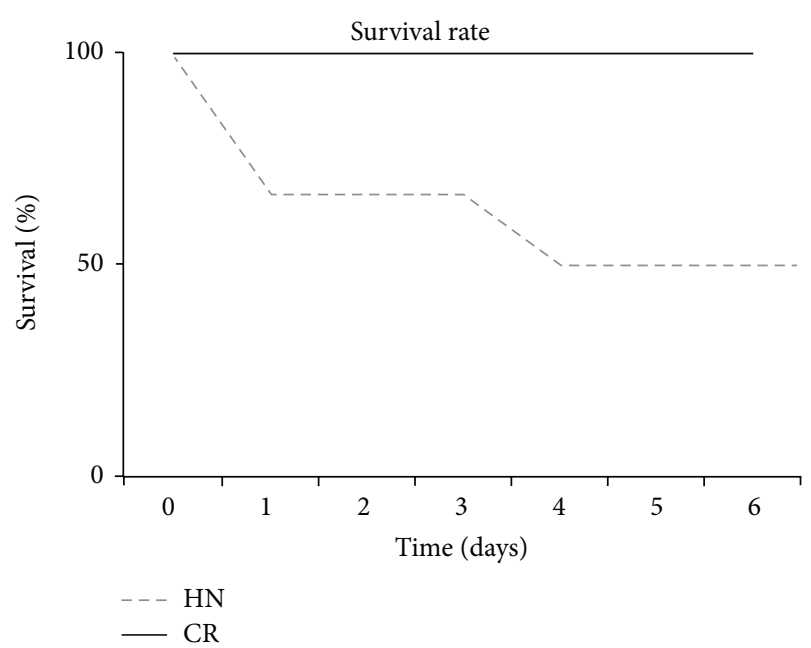

FIGURE 2: Survival rates between rats supplied by Harlan and Charles River.

severity of injury is dependent on the method of occlusion. Subcortical regions are supplied by the lateral striate arteries branching from the middle cerebral artery (MCA) [6]. It has been previously shown that subcortical lesion differences in a filament model of ischemia in rats from different vendors were not as pronounced as cortical lesion differences [24]. If differences between lesion sizes were to be observed in the cortex they would likely be due to the difference in collateral circulation between strains and/or vendors.

The current study showed that in the first 3 hours after the two-vessel occlusion, Sprague-Dawley rats supplied by Charles River had greater blood flow in the stroke lesion compared to rats supplied by Harlan. Specifically, the Harlan animals showed a $67 \pm 6 \%$ decrease compared to a $37 \pm$ $4 \%$ decrease in Charles River rats. Similarly, Marosi et al. [22] compared the acute effects of bilateral carotid occlusion induced ischemia on Wistar rats supplied by Charles River and Harlan. In their acute electrophysiological experiments based on hippocampal CA1 responses they showed that the Charles River rats immediately compensated the two-vessel occlusion more effectively compared to the Harlan rats. At three days after ischemia, the differences were not present. Prieto et al. [9] also reported major differences in blood flow measured by Laser Doppler flowmetry in Sprague-Dawley, Long-Evans, and Wistar Rats. They reported decreases in local cerebral blood flow during the first 20 minutes of occlusion significantly greater in Long-Evans $(74.6 \pm 8.9 \%)$ than in Sprague-Dawley $(50.7 \pm 26.8 \%)$ and Wistar $(49.0 \pm 21.9 \%)$ rats. Their results imply that the intraluminal MCAO model produced more pronounced, persistent, and stable blood flow reduction with less internal variability in the Long-Evans rats compared to the Sprague-Dawley or Wistar rats.

Paradoxically, MR images acquired one day after ischemia showed significantly larger lesion volume in rats supplied by Charles River compared to rats from Harlan. The significance remained at six days after ischemia although this comparison included only three rats supplied by Harlan due to their increased mortality rate. On day six, the size of the lesion 

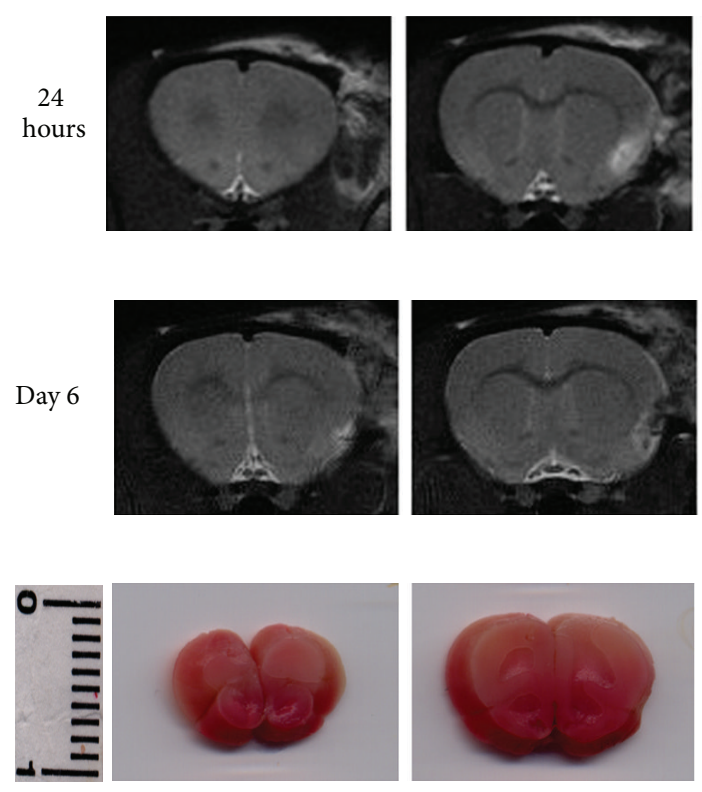
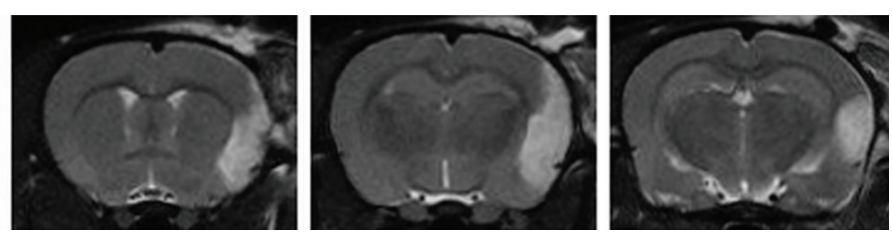

(a)
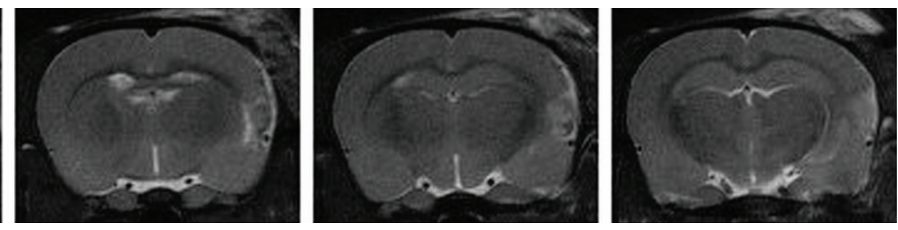

(b)
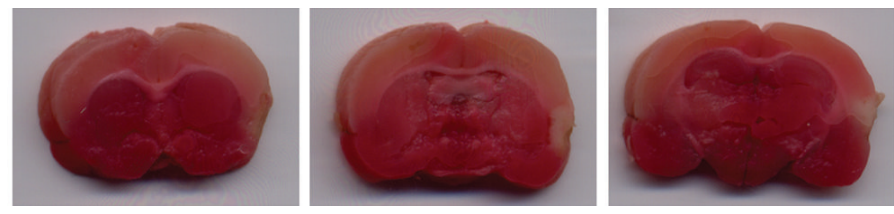

(c)

Figure 3: Ischemic damage in a representative CR animal at day 1 (a), day 6 (b), and final histology (c). Normal tissue is pink in TTC while ischemic tissue is white.

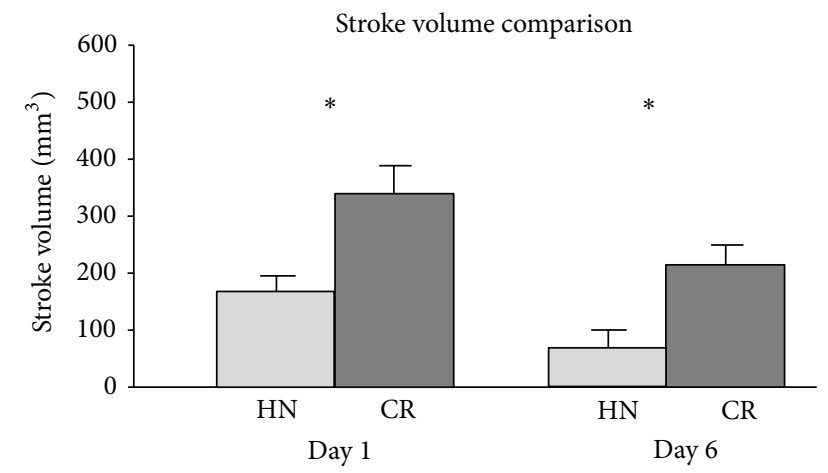

FIGURE 4: Average stroke volume at day 1 and day 6 after occlusion in animals supplied by Harlan and Charles River. Significant differences are observed between suppliers at 1 day and 6 days $(P=0.04$ and $P=0.02$, resp., 2 tailed unpaired $t$-test).

on TTC stained slices also varied in size between the Harlan and Charles River rats. Higher mortality rates and more pronounced behavioral deficits were observed in the Harlan animals. A similar study by Oliff et al. [24] compared Sprague-Dawley (SD) rats from Simonsen, Charles River, and Taconic Labs following ischemia (filament model of MCAO). This study showed that after MCAO, infarct volumes in rats supplied by Simonsen were two- to sixteen-fold smaller than the lesions observed in rats supplied by Taconic animals. Despite a smaller lesion volume in rats supplied by Harlan in the current study, these animals may have suffered a more severe insult due to poorer collateral supply compared to the Charles River animals [27].
There are several limitations to the current study. First, the number of animals from each supplier was relatively small. Future studies should examine a larger cohort to validate the current findings. Second, blood flow measurements were made from tissue regions that were manually outlined using the MRI images as a guide. Future studies should register the MR images to the CT perfusion maps to ensure that the entire region of tissue damage is sampled. The strengths of the current study include careful control of the temperature of all animals within the range of $36.5-37.5^{\circ} \mathrm{C}$. This effort was necessary because it is well known that hypothermia causes neuroprotection and can lead to reduction of lesion size in animal models of stroke [41]. All animals were exposed to the same diet to reduce variations that could also result in differential response to ischemia.

The current study is the first to compare the effects of a three-vessel occlusion model of stroke in Sprague-Dawley rats from different suppliers combining in vivo measures of lesion volume, cerebral perfusion, and motor function in the same animals. The results provide additional evidence that vendor differences should be an important consideration when animals are selected for various research models.

Rats supplied by Harlan had lower blood flow, increased behavioural deficits, and increased mortality despite having smaller lesions compared to rats from Charles River. These marked interstrain differences could provide an interesting tool in the study of the impact of genetic and environmental factors on ischemia and long term outcome.

\section{Conflict of Interests}

The authors declare that there is no conflict of interests regarding the publication of this paper. 


\section{Acknowledgments}

Funding was provided by the Heart and Stroke Foundation of Canada, the Canadian Institutes of Health Research (MME15594) and the Ivey BMO-Financial Group Scientist in Brain Disorders Imaging Award. We would like to thank our veterinary technician, Miranda Bellyou-Camillery, for preparing the animals and performing the surgeries.

\section{References}

[1] J. Agulla, B. Argibay, M. Pérez-Mato, D. Brea, P. RamosCabrer, and J. Castillo, "Comparison of the lesion produced by permanent focal cerebral ischaemia in three animal models using magnetic resonance imaging," Revista de Neurologia, vol. 53, no. 5, pp. 265-274, 2011.

[2] M. Bacigaluppi, G. Comi, and D. M. Hermann, "Animal models of ischemic stroke. Part two: modeling cerebral ischemia," The Open Neurology Journal, vol. 4, pp. 34-38, 2010.

[3] J. B. Casals, N. C. G. Pieri, M. L. T. Feitosa et al., "The use of animal models for stroke research: a review," Comparative Medicine, vol. 61, no. 4, pp. 305-313, 2011.

[4] M. J. O'Neill and J. A. Clemens, "Rodent models of global cerebral ischemia," in Current Protocols in Neuroscience, chapter 9, unit 9.6, John Wiley \& Sons, New York, NY, USA, 2001.

[5] K. M. Sicard and M. Fisher, "Animal models of focal brain ischemia," Experimental \& Translational Stroke Medicine, vol. 1, article 7, 2009.

[6] A. Tamura, D. I. Graham, J. McCulloch, and G. M. Teasdale, "Focal cerebral ischaemia in the rat: 1. Description of technique and early neuropathological consequences following middle cerebral artery occlusion," Journal of Cerebral Blood Flow and Metabolism, vol. 1, no. 1, pp. 53-60, 1981.

[7] A. Tamura, D. I. Graham, J. McCulloch, and G. M. Teasdale, "Focal cerebral ischaemia in the rat: 2. Regional cerebral blood flow determined by [14C]iodoantipyrine autoradiography following middle cerebral artery occlusion," Journal of Cerebral Blood Flow and Metabolism, vol. 1, no. 1, pp. 61-69, 1981.

[8] H. Nagasawa and K. Kogure, "Correlation between cerebral blood flow and histologic changes in a new rat model of middle cerebral artery occlusion," Stroke, vol. 20, no. 8, pp. 1037-1043, 1989.

[9] R. Prieto, F. Carceller, J. M. Roda, and C. Avendaño, “The intraluminal thread model revisited: rat strain differences in local cerebral blood flow," Neurological Research, vol. 27, no. 1, pp. 4752, 2005.

[10] T. Shigeno, G. M. Teasdale, J. McCulloch, and D. I. Graham, "Recirculation model following MCA occlusion in rats. Cerebral blood flow, cerebrovascular permeability, and brain edema," Journal of Neurosurgery, vol. 63, no. 2, pp. 272-277, 1985.

[11] N. Shimamura, N. Matsuda, K. Katayama, and H. Ohkuma, "Novel rat middle cerebral artery occlusion model: transfemoral artery approach combined with preservation of the external carotid artery," Journal of Neuroscience Methods, vol. 184, no. 2, pp. 195-198, 2009.

[12] H. Yanamoto, I. Nagata, N. Hashimoto, and H. Kikuchi, "Threevessel occlusion using a micro-clip for the proximal left middle cerebral artery produces a reliable neocortical infarct in rats," Brain Research Protocols, vol. 3, no. 2, pp. 209-220, 1998.

[13] C. Backhauss, C. Karkoutly, M. Welsch, and J. Krieglstein, "A mouse model of focal cerebral ischemia for screening neuroprotective drug effects," Journal of Pharmacological and Toxicological Methods, vol. 27, no. 1, pp. 27-32, 1992.

[14] T. Shigeno, J. McCulloch, D. I. Graham, A. D. Mendelow, and G. M. Teasdale, "Pure cortical ischemia versus striatal ischemia. Circulatory, metabolic, and neuropathologic consequences," Surgical Neurology, vol. 24, no. 1, pp. 47-51, 1985.

[15] M. D. Ginsberg and R. Busto, "Rodent models of cerebral ischemia," Stroke, vol. 20, no. 12, pp. 1627-1642, 1989.

[16] K. Griffin, A. Polichnowski, H. Licea-Vargas et al., "Large BPdependent and -independent differences in susceptibility to nephropathy after nitric oxide inhibition in Sprague-Dawley rats from two major suppliers," The American Journal of Physiology, vol. 302, no. 1, pp. 173-182, 2012.

[17] D. M. Pollock and A. Rekito, "Hypertensive response to chronic NO synthase inhibition is different in Sprague-Dawley rats from two suppliers," The American Journal of Physiology, vol. 275, no. 5, pp. 1719-1723, 1998.

[18] M. Langer, C. Brandt, and W. Löscher, "Marked strain and substrain differences in induction of status epilepticus and subsequent development of neurodegeneration, epilepsy, and behavioral alterations in rats," Epilepsy Research, vol. 96, no. 3, pp. 207-224, 2011.

[19] E. G. Deune and R. K. Khouri, "Rat strain differences in flap tolerance to ischemia," Microsurgery, vol. 16, no. 11, pp. 765-767, 1995.

[20] D. Duverger and E. T. MacKenzie, "The quantification of cerebral infarction following focal ischemia in the rat: influence of strain, arterial pressure, blood glucose concentration, and age," Journal of Cerebral Blood Flow and Metabolism, vol. 8, no. 4, pp. 449-461, 1988.

[21] S. Kacew, R. Dixit, and Z. Ruben, "Diet and rat strain as factors in nervous system function and influence of confounders," Biomedical and Environmental Sciences, vol. 11, no. 3, pp. 203217, 1998.

[22] M. Marosi, G. Rákos, H. Robotka et al., "Hippocampal (CA1) activities in Wistar rats from different vendors. Fundamental differences in acute ischemia," Journal of Neuroscience Methods, vol. 156, no. 1-2, pp. 231-235, 2006.

[23] V. C. Moser, K. L. McDaniel, and P. M. Phillips, "Rat strain and stock comparisons using a functional observational battery: baseline values and effects of amitraz," Toxicology and Applied Pharmacology, vol. 108, no. 2, pp. 267-283, 1991.

[24] H. S. Oliff, E. Weber, G. Eilon, and P. Marek, "The role of strain/ vendor differences on the outcome of focal ischemia induced by intraluminal middle cerebral artery occlusion in the rat," Brain Research, vol. 675, no. 1-2, pp. 20-26, 1995.

[25] H. S. Oliff, E. Weber, B. Miyazaki, and P. Marek, "Infarct volume varies with rat strain and vendor in focal cerebral ischemia induced by transcranial middle cerebral artery occlusion," Brain Research, vol. 699, no. 2, pp. 329-331, 1995.

[26] S. Palm, E. Roman, and I. Nylander, "Differences in voluntary ethanol consumption in Wistar rats from five different suppliers," Alcohol, vol. 45, no. 6, pp. 607-614, 2011.

[27] H. S. Oliff, P. Coyle, and E. Weber, "Rat strain and vendor differences in collateral anastomoses," Journal of Cerebral Blood Flow and Metabolism, vol. 17, no. 5, pp. 571-576, 1997.

[28] A. Cenic, D. G. Nabavi, R. A. Craen, A. W. Gelb, and T.-Y. Lee, "Dynamic CT measurement of cerebral blood flow: a validation study," The American Journal of Neuroradiology, vol. 20, no. 1, pp. 63-73, 1999. 
[29] J. Biernaskie, D. Corbett, J. Peeling, J. Wells, and H. Lei, "A serial MR study of cerebral blood flow changes and lesion development following endothelin-1-induced ischemia in rats," Magnetic Resonance in Medicine, vol. 46, no. 4, pp. 827-830, 2001.

[30] M. Modo, R. P. Stroemer, E. Tang, T. Veizovic, P. Sowniski, and H. Hodges, "Neurological sequelae and long-term behavioural assessment of rats with transient middle cerebral artery occlusion," Journal of Neuroscience Methods, vol. 104, no. 1, pp. 99$109,2000$.

[31] C. P. Montoya, L. J. Campbell-Hope, K. D. Pemberton, and S. B. Dunnett, "The "staircase test": a measure of independent forelimb reaching and grasping abilities in rats," Journal of Neuroscience Methods, vol. 36, no. 2-3, pp. 219-228, 1991.

[32] T. Schallert, S. M. Fleming, J. L. Leasure, J. L. Tillerson, and S. T. Bland, "CNS plasticity and assessment of forelimb sensorimotor outcome in unilateral rat models of stroke, cortical ablation, parkinsonism and spinal cord injury," Neuropharmacology, vol. 39, no. 5, pp. 777-787, 2000.

[33] T. Schallert, J. L. Leasure, and B. Kolb, "Experience-associated structural events, subependymal cellular proliferative activity, and functional recovery after injury to the central nervous system," Journal of Cerebral Blood Flow and Metabolism, vol. 20, no. 11, pp. 1513-1528, 2000.

[34] V. Windle, A. Szymanska, S. Granter-Button et al., "An analysis of four different methods of producing focal cerebral ischemia with endothelin-1 in the rat," Experimental Neurology, vol. 201, no. 2, pp. 324-334, 2006.

[35] M. D. Lindner, V. K. Gribkoff, N. A. Donlan, and T. A. Jones, "Long-lasting functional disabilities in middle-aged rats with small cerebral infarcts," The Journal of Neuroscience, vol. 23, no. 34, pp. 10913-10922, 2003.

[36] T. M. Barth and B. B. Stanfield, "The recovery of forelimbplacing behavior in rats with neonatal unilateral cortical damage involves the remaining hemisphere," Journal of Neuroscience, vol. 10, no. 10, pp. 3449-3459, 1990.

[37] T. Schallert, D. A. Kozlowski, J. L. Humm, and R. R. Cocke, "Use-dependent structural events in recovery of function," Advances in Neurology, vol. 73, pp. 229-238, 1997.

[38] T. Schallert and I. Q. Whishaw, "Bilateral cutaneous stimulation of the somatosensory system in hemidecorticate rats," Behavioral Neuroscience, vol. 98, no. 3, pp. 518-540, 1984.

[39] J. B. Bederson, L. H. Pitts, M. Tsuji, M. C. Nishimura, R. L. Davis, and H. Bartkowski, "Rat middle cerebral artery occlusion: evaluation of the model and development of a neurologic examination," Stroke, vol. 17, no. 3, pp. 472-476, 1986.

[40] J. B. Bederson, L. H. Pitts, S. M. Germano, M. C. Nishimura, R. L. Davis, and H. M. Bartkowski, "Evaluation of 2,3,5-triphenyltetrazolium chloride as a stain for detection and quantification of experimental cerebral infarction in rats," Stroke, vol. 17, no. 6, pp. 1304-1308, 1986.

[41] D. Xue, Z.-G. Huang, K. E. Smith, and A. M. Buchan, "Immediate or delayed mild hypothermia prevents focal cerebral infection," Brain Research, vol. 587, no. 1, pp. 66-72, 1992. 


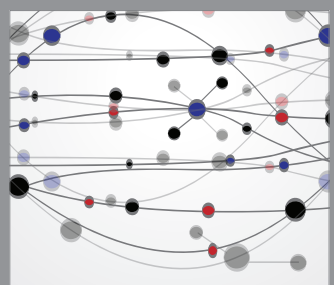

The Scientific World Journal
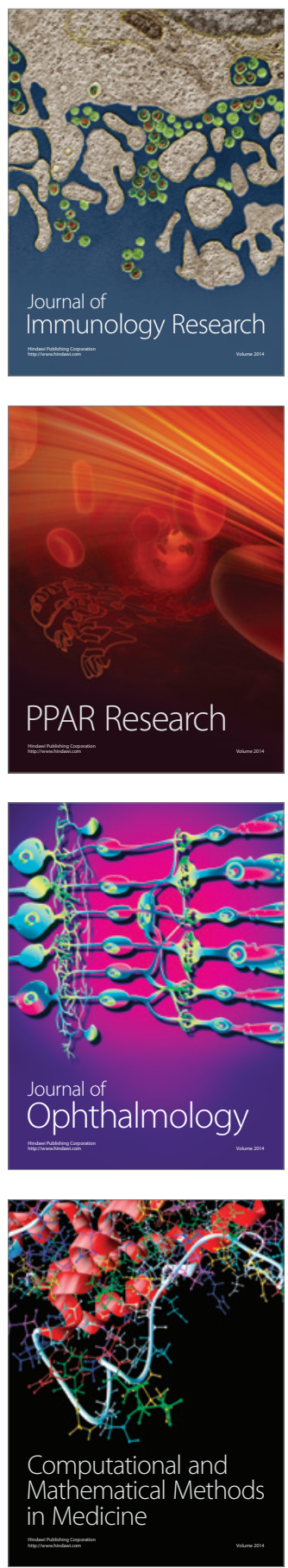

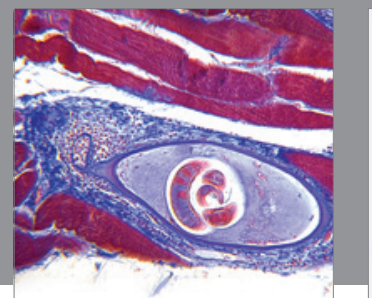

Gastroenterology

Research and Practice
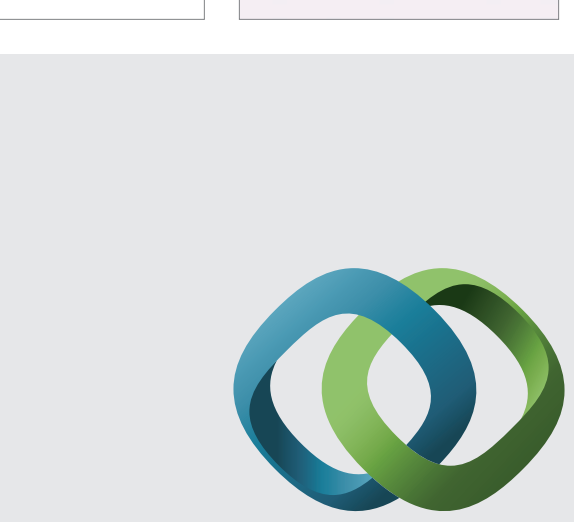

\section{Hindawi}

Submit your manuscripts at

http://www.hindawi.com
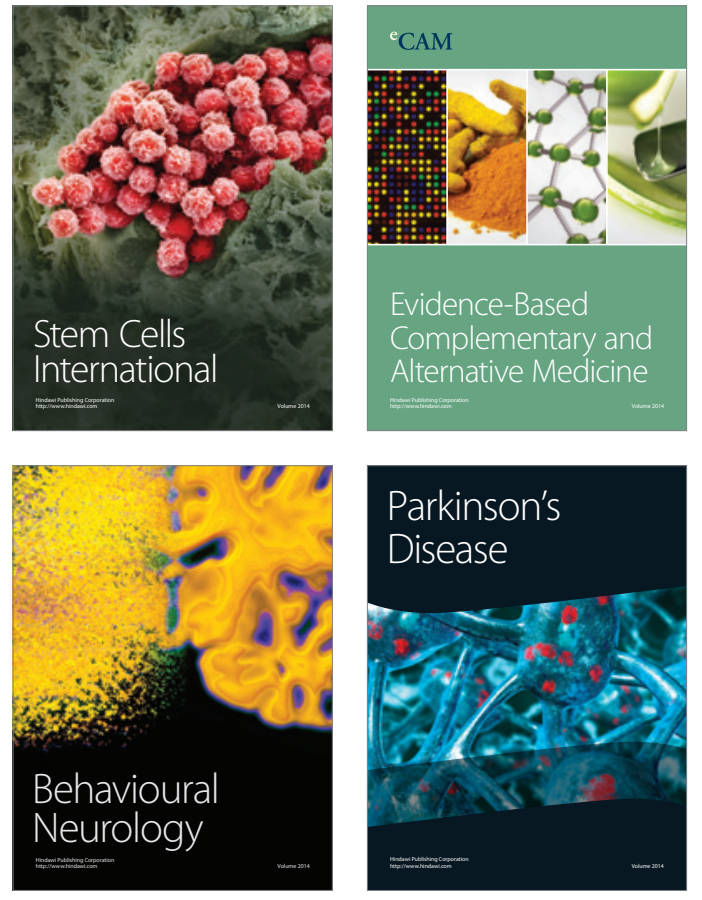
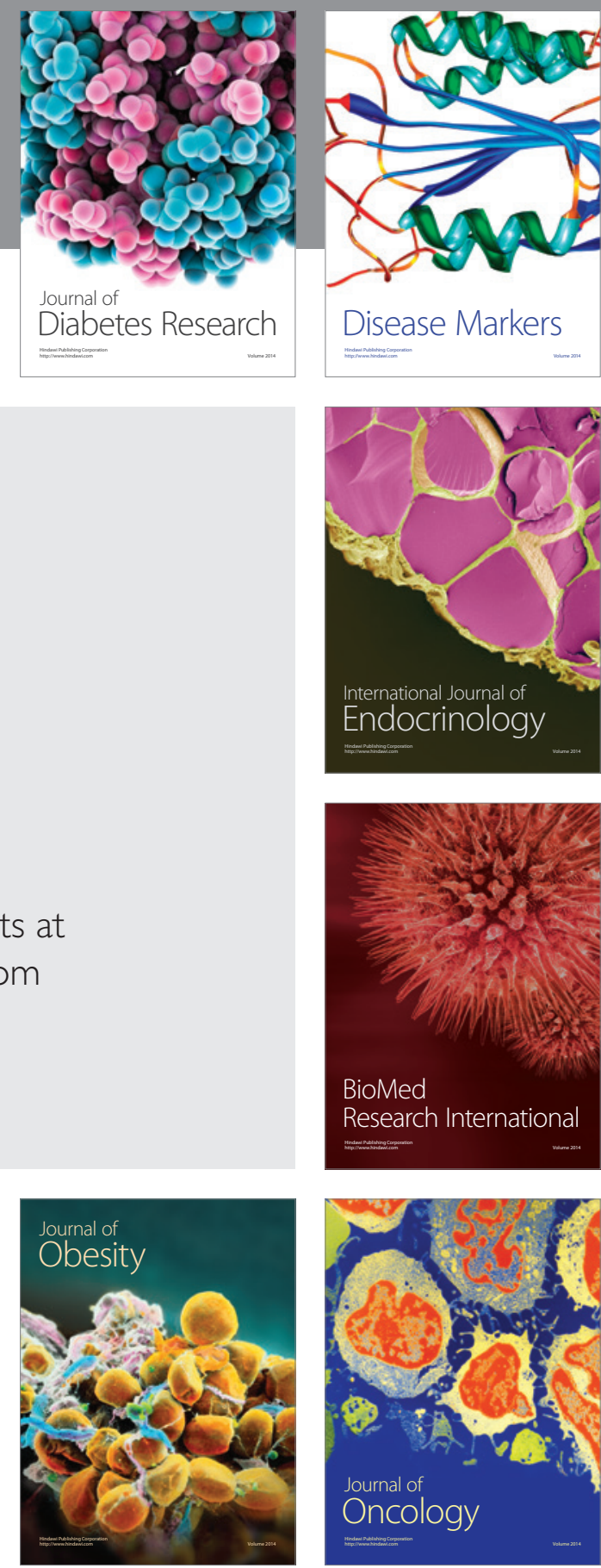

Disease Markers
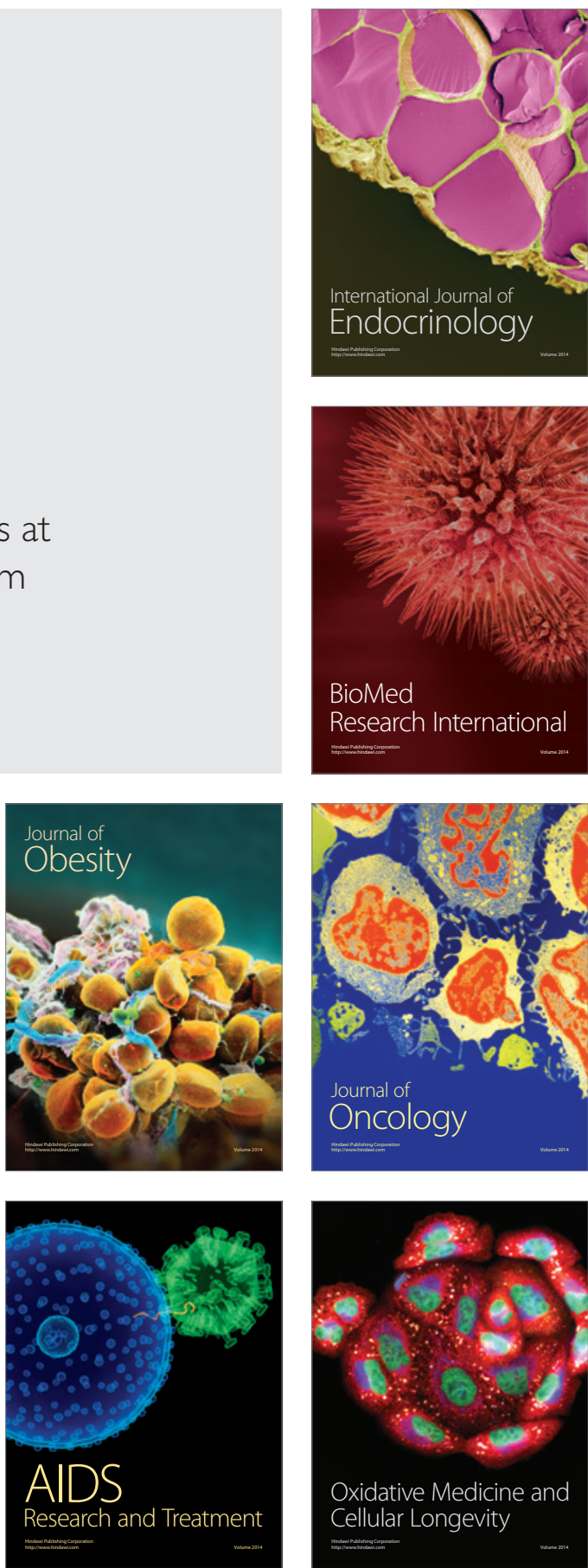